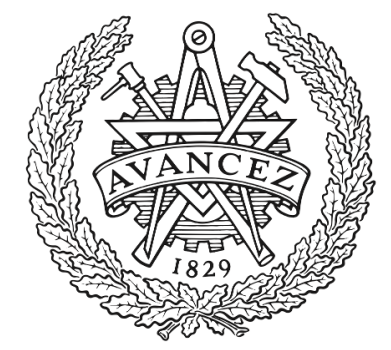

CHALMERS

UNIVERSITY OF TECHNOLOGY

\title{
Antialiased transmitter-side digital backpropagation
}

Downloaded from: https://research.chalmers.se, 2023-04-26 08:12 UTC

Citation for the original published paper (version of record):

Li, S., Karlsson, M., Agrell, E. (2020). Antialiased transmitter-side digital backpropagation. IEEE

Photonics Technology Letters, 32(18): 1211-1214. http://dx.doi.org/10.1109/LPT.2020.3017228

N.B. When citing this work, cite the original published paper.

(C)2020 IEEE. Personal use of this material is permitted.

However, permission to reprint/republish this material for advertising or promotional purposes 


\title{
Antialiased Transmitter-Side Digital Backpropagation
}

\author{
Shen $\mathrm{Li}^{\circledR}$, Magnus Karlsson ${ }^{\circledR}$, Senior Member, IEEE, and Erik Agrell ${ }^{\circledR}$, Fellow, IEEE
}

\begin{abstract}
Digital backpropagation (DBP) is an electronic scheme for compensating nonlinear distortions in fiber transmission systems. Due to the nonlinearity-induced spectral broadening, the data must be oversampled to avoid aliasing, which increases the complexity and power consumption of the scheme. In this work, we show that aliasing can alternatively be prevented by distributed antialiasing filters, at a lower complexity. We proposed a new modified split-step Fourier method (SSFM) with easy-to-implement low-pass filters (LPFs) in the linear steps to avoid aliasing due to spectral broadening. Both the forward fiber propagation and a transmitter-side DBP are simulated using the modified SSFM. High-order modulation formats such as 256-ary quadrature-amplitude-modulation (256-QAM) and 1024-QAM transmissions at 28 Gbaud and 64 Gbaud over $1000 \mathrm{~km}$ fiber are considered, and our results show that the complexity of the DBP can be reduced by up to $50 \%$. The optimal bandwidth of the LPFs is studied for both forward propagation and the DBP.
\end{abstract}

Index Terms-Digital backpropagation, split-step Fourier method, antialiasing, low-pass filter, dispersion and nonlinear impairments compensation, oversampling rate.

\section{INTRODUCTION}

$\mathbf{T}$ HE split-step Fourier method (SSFM) is a numerical method to simulate the signal propagation along the nonlinear optical fiber. The SSFM not only can be used for modeling fiber propagation, but also is an effective technique for compensating the linear and nonlinear impairments of fiber transmission, which is widely known as digital backpropagation (DBP). DBP can be implemented either at the transmitter side to precompensate the impairments of the fiber link before signal transmission [1]-[4], or at the receiver side to undo channel distortion after transmission [5]-[11], or both at the transmitter and the receiver sides [12].

However, the main drawback of the SSFM/DBP is the high cost of complexity, which makes it hard to implement in reality and time-consuming for simulation. The computational complexity of the SSFM/DBP is proportional to the oversampling rate of the signal (a multiple of the baud rate), and the number of spatial steps. Much work has been done to reduce the complexity of both the forward SSFM [13]-[18] and the

Manuscript received July 2, 2020; revised July 30, 2020; accepted August 7, 2020. Date of publication August 17, 2020; date of current version August 27, 2020. This work was supported by the Swedish Research Council (VR) under Grant 2017-03702. (Corresponding author: Shen Li.)

Shen Li and Erik Agrell are with the Department of Electrical Engineering, Chalmers University of Technology, 41296 Gothenburg, Sweden (e-mail: shenl@chalmers.se).

Magnus Karlsson is with the Department of Microtechnology and Nanoscience, Chalmers University of Technology, 41296 Gothenburg, Sweden.

Color versions of one or more of the figures in this letter are available online at http://ieeexplore.iee.org.

Digital Object Identifier 10.1109/LPT.2020.3017228
DBP [7]-[11]. In [7], [13]-[17], various step size selection schemes and optimization rules are studied. In [18], the complexity of the SSFM was reduced by replacing the linear frequency domain step and the successive Fourier transforms with an optimized time-domain filter. In [8], [9], low-pass filters (LPFs) are used to filter the nonlinear steps of the DBP (the intensity waveform used to calculate the nonlinear phase modulator), which leads to reduction of the required oversampling rate and number of steps. In [10], [11], the complexity were reduced by replacing the traditional linear operator of the DBP with learned short and symmetric finite impulse response filters. Nevertheless, aliasing of the spectrum, which might happen during the process of the SSFM/DBP due to spectral broadening, has never been avoided in the previous research, apart from increasing the oversampling rate which adds complexity.

In order to avoid aliasing to maintain the signal quality without additional complexity, we investigate a modified SSFM algorithm by replacing the standard all-pass fitler with a low-pass filter (LPF) in each linear step in the frequency domain. Similar LPFs were proposed in [19] to reduce channel crosstalk in wavelength-multiplexed systems. In contrast to [19], we do not propose to physically modify the propagation channel-our aim is instead to simulate the unmodified nonlinear propagation channel with improved accuracy. Our approach is also different from [8], [9], where LPFs were applied in the nonlinear step of the SSFM. The LPFs can be implemented at no added complexity-in fact, the complexity is slightly reduced, because the LPFs have fewer nonzero coefficients than the regular all-pass filters that otherwise build up the linear steps. Just as with the traditional SSFM, the filter coefficients can be precomputed and stored.

Our simulations show that with these LPFs, the simulation accuracy is improved given a fixed simulation complexity [20]. In this letter, we extend our work in [20] and use the modified SSFM to precompensate the fiber impairments before transmission (performing a transmitter-side DBP (TS-DBP)). This filtering method achieves more effective compensation, i.e., the bit error rate (BER) is reduced for a given DBP complexity. The optimal bandwidth of the LPF is also analysed for some high-order modulation formats.

\section{SySTEM MODEL}

\section{A. Forward SSFM for Modelling the Channel}

Let $\mathbf{a}(t, z)=\left[a_{x}(t, Z) a_{y}(t, Z)\right]^{\mathrm{T}}$ be the electrical field of $\mathrm{X}$ and $\mathrm{Y}$ polarizations in complex baseband propagating along the fiber at time $t$ and distance $z$. The Manakov equation for 


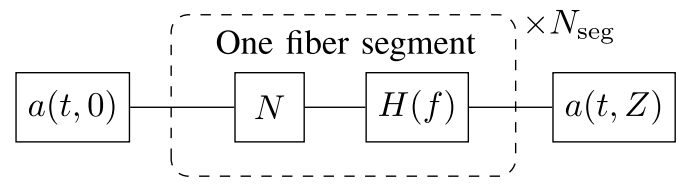

Fig. 1. The improved SSFM structure with LPFs for modeling fiber propagation.

the evolution of $\mathbf{a}(t, z)$ in an unamplified fiber span can be written as

$$
\begin{aligned}
\frac{\partial \mathbf{a}(t, z)}{\partial z}= & -\frac{\alpha-g(z)}{2} \mathbf{a}(t, z)-j \frac{\beta_{2}}{2} \frac{\partial^{2} \mathbf{a}(t, z)}{\partial t^{2}} \\
& +j \gamma \frac{8}{9}\|\mathbf{a}(t, z)\|^{2} \mathbf{a}(t, z)+\mathbf{n}(t, z)
\end{aligned}
$$

where $\mathbf{a}(t, 0)$ is the input signal, $\alpha$ is the attenuation factor, $\beta_{2}$ is the dispersion parameter, and $\gamma$ is the nonlinear parameter, $g(z)$ is the gain profile function, and $\mathbf{n}(t, z)$ is the amplified spontaneous emission (ASE) noise. Here we consider the two-polarization transmission governed by the Manakov equation. However, this filtering method also works for the scalar nonlinear Schrödinger equation. The SSFM discretizes the transmission distance $Z$ into $N_{\text {seg }}$ spatial steps in which a linear operator $-\frac{\alpha}{2}-j \frac{\beta_{2}}{2} \frac{\partial^{2}}{\partial t^{2}}$ and a nonlinear operator $N=j \gamma \frac{8}{9}\|\mathbf{a}(t, z)\|^{2}$ can be applied separately in each step. We modify the linear step by replacing the standard all-pass filter with an LPF with bandwidth $W$, as shown in Fig. 1. The proposed linear step is a multiplication with

$$
H(f)= \begin{cases}\exp \left(-\alpha \Delta z_{1}+2 j \pi^{2} f^{2} \beta_{2} \Delta z_{1}\right), & |f| \leq W \\ 0, & W<|f| \leq \frac{W_{\mathrm{s}}}{2}\end{cases}
$$

in the frequency domain, where $f$ is the frequency component of the signal, $\Delta z_{1}=Z / N_{\text {seg }}$ is the step size, $W_{\mathrm{s}}$ is the sampling rate and the filter bandwidth $W \leq W_{\mathrm{s}} / 2$. When $W=W_{\mathrm{s}} / 2, H(f)$ represents an all-pass filter, which is the linear step in traditional SSFM. Thus, since fewer complex exponentials and multiplications need to be computed when $W<W_{\mathrm{s}} / 2$, the proposed scheme has slightly lower complexity than the traditional one. There will be power loss since the LPF filters out some part of the signal in each step. These lost frequency components are exactly the parts that would otherwise be transferred to the wrong frequencies during spectral broadening and cause aliasing, so it is beneficial to lose some of the signal power. However, the LPF bandwidth $W$ intuitively cannot be too narrow to avoid erasing too much information of the signal.

\section{B. Transmitter-Side Precompensation}

The SSFM can also be used for compensating the nonlinear and linear impairments by solving the inverse Manakov equation. The DBP uses $K_{\mathrm{cps}}$ compensation steps in each of which the nonlinear compensator $N^{-1}=-j \gamma \frac{8}{9}\|\mathbf{a}(t, z)\|^{2}$ and the linear compensator $\frac{\alpha}{2}+j \frac{\beta_{2}}{2} \frac{\partial^{2}}{\partial t^{2}}$ are performed separately. The proposed transmitter-side DBP structure is shown in Fig. 2,

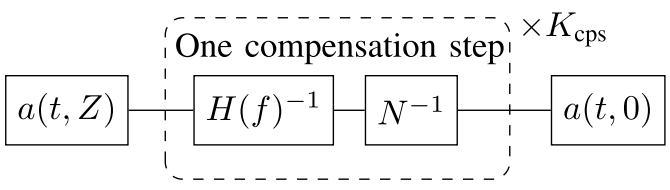

Fig. 2. The improved transmiter-side DBP with LPFs for precompensating transmission impairments.

where $H(f)^{-1}$ is the low-passed linear compensator

$$
H(f)^{-1}= \begin{cases}\exp \left(\alpha \Delta z_{2}-2 j \pi^{2} f^{2} \beta_{2} \Delta z_{2}\right), & |f| \leq W \\ 0, & W<|f| \leq \frac{W_{\mathrm{s}}}{2}\end{cases}
$$

where the compensation step length $\Delta z_{2}=Z / K_{c p s}$, together with the oversampling rate, determines the DBP complexity.

\section{ImProved SimUlation ACCURACY}

We consider 16-ary quadrature amplitude modulation (16-QAM) transmitted at 28 Gbaud and 64 Gbaud through single-mode fiber using a root-raised-cosine pulse with roll-off factor $10 \%$ and ideal distributed amplification [24, pp. 69-70] which compensates the attenuation. The fiber parameters are $\beta_{2}=-21.7 \mathrm{ps}^{2} / \mathrm{km}$ and $\gamma=1.27(\mathrm{~W} \cdot \mathrm{km})^{-1}, g(z)=\alpha=$ $0.2 \mathrm{~dB} / \mathrm{km}$, and amplifier noise is neglected. The need for small enough time resolution $\Delta t$ for simulating the nonlinear fiber propagation is illustrated in Fig. 3, where $T_{\mathrm{S}}$ is the symbol time [21], [22]. In this example, the SSFM output in X polarization $\hat{a}_{x}(t, Z)$ converges to the output of the Manakov equation $a_{x}(t, Z)$ with 30 samples per symbol (the black curve in Fig. 3). As $\Delta t$ increases, $\hat{a}_{x}(t, Z)$ becomes increasingly deviated from $a_{x}(t, Z)$. After increasing $\Delta t$ to $T_{\mathrm{s}} / 4, \hat{a}_{x}(t, Z)$ is completely independent of $a_{x}(t, Z)$. This phenomenon is caused by aliasing resulting from spectral broadening [23]. With LPFs, all the outputs with different $\Delta t$ become closer to $a_{x}(t, Z)$, provided that $W$ is carefully selected and optimized.

To evaluate the simulation error, we define the normalized squared difference (NSD)

$$
\mathrm{NSD}=\frac{\int\|\mathbf{a}(t, Z)-\hat{\mathbf{a}}(t, Z)\|^{2} d t}{\int\|\mathbf{a}(t, Z)\|^{2} d t}
$$

between the converged Manakov output $\mathbf{a}(t, Z)$ (without LPFs) and another simulated output $\hat{\mathbf{a}}(t, Z)$. Let $\Delta t_{\mathrm{M}}=T_{\mathrm{s}} / 30$ and $\Delta z_{\mathrm{M}}=0.02 \mathrm{~km}$ denote the time discretization and step size to simulate $\mathbf{a}(t, Z)$. It was numerically validated that in all cases within this section, these parameters are sufficient for the SSFM to converge to $\mathbf{a}(t, Z)$. The relative filter bandwidth $W /\left(W_{\mathrm{s}} / 2\right)$ for some example cases is analyzed in Fig. 4. Under such high launch power, the transmission at 28 Gbaud needs larger oversampling rates (more spectral extension) to avoid aliasing. The minimum NSD appears when $W /\left(W_{\mathrm{s}} / 2\right)$ is between $70 \%$ and $90 \%$, while $100 \%$ means the SSFM without LPFs. The power loss for all cases with optimized $W$ in Fig. 4 is less than $2.5 \%$. 

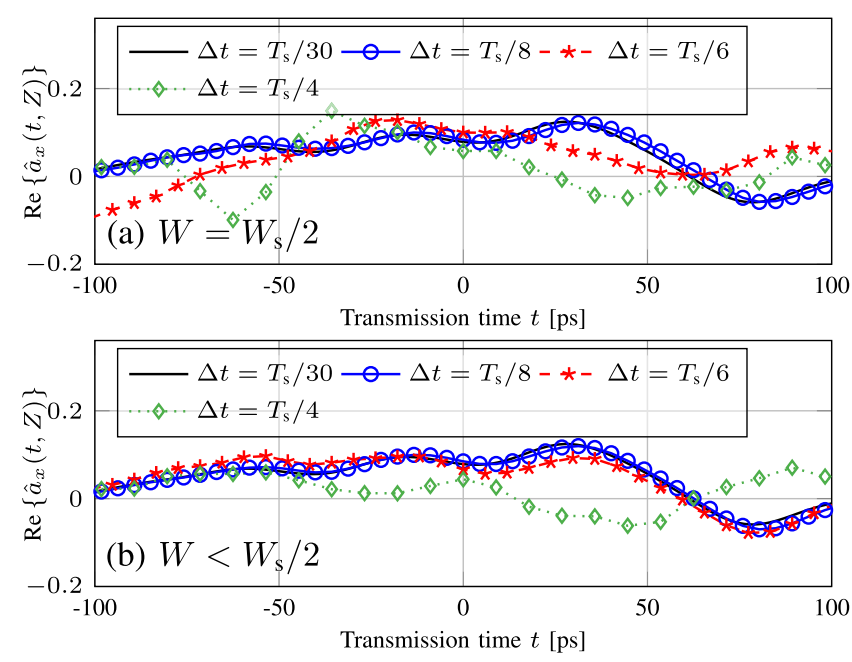

Fig. 3. The SSFM output with different $\Delta t$ becomes more similar to the converged output of the Manakov using LPFs. Parameters: 28 Gbaud symbol rate; $P=15 \mathrm{dBm} ; Z=1000 \mathrm{~km} ; \Delta z_{1}=0.02 \mathrm{~km}$. In case (b), the LPF bandwidth $W$ is optimized for each $\Delta t$.

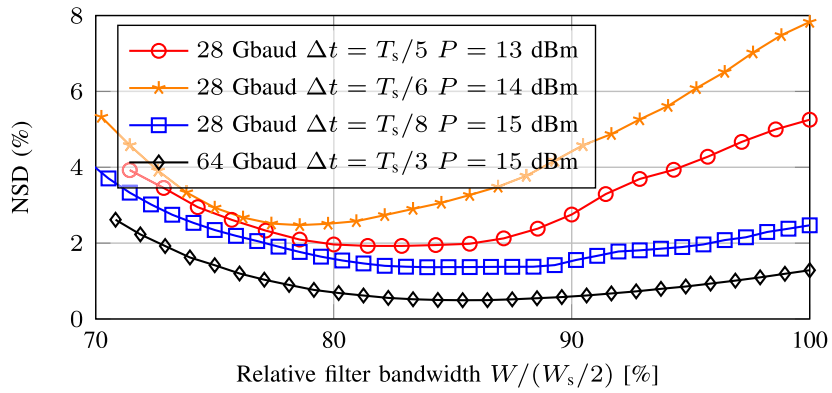

Fig. 4. The NSD is improved with the LPFs and the optimal relative filter bandwidth appears at $70 \%-90 \%$. Parameters: $Z=1000 \mathrm{~km}$. The right-hand edge $(100 \%)$ represents the NSD with the traditional SSFM.

\section{Reduced COMPlexity Digital BACKPROPAGATION}

We consider 256-QAM and 1024-QAM with Gray mapping transmitted at 28 and 64 Gbaud through $1000 \mathrm{~km}$ single-mode fiber using a root-raised-cosine pulse with roll-off factor $10 \%$ and ideal distributed amplification which compensates the attenuation, i.e., $g(z)=\alpha$. The fiber parameters $\alpha, \beta_{2}$ and $\gamma$ are the same as in Section III. To simulate the fiber channel, we use traditional SSFM and choose the pretested converging parameters $\Delta z_{\mathrm{M}}=2 \mathrm{~km}$ and $\Delta t_{\mathrm{M}}=T_{s} / 30$ for all cases. The ASE noise power of each step is $2 \alpha \Delta z_{\mathrm{M}} K_{\mathrm{T}} h v W_{\mathrm{s}}$ [25], where the photon occupancy factor $K_{\mathrm{T}}$ is 1.13 , and the photon energy is $h v=1.28 \cdot 10^{-19} \mathrm{~J}$. We simulate a system with TS-DBP, since our purpose is to reduce the signal distortion caused by aliasing resulting from spectral broadening. With receiver-side DBP, aliasing happens during the simulation of fiber propagation, but the DBP after is a process of spectral narrowing, in which LPFs cannot help too much.

With TS-DBP, we compare the BER as a function of the input power $P$ for different modulation formats, baud rates, and oversampling rates in the DBP as shown in Fig. 5. For 256-QAM transmission at 28 Gbaud with 2 samples per symbol, with our LPFs, the minimum BER is reduced from

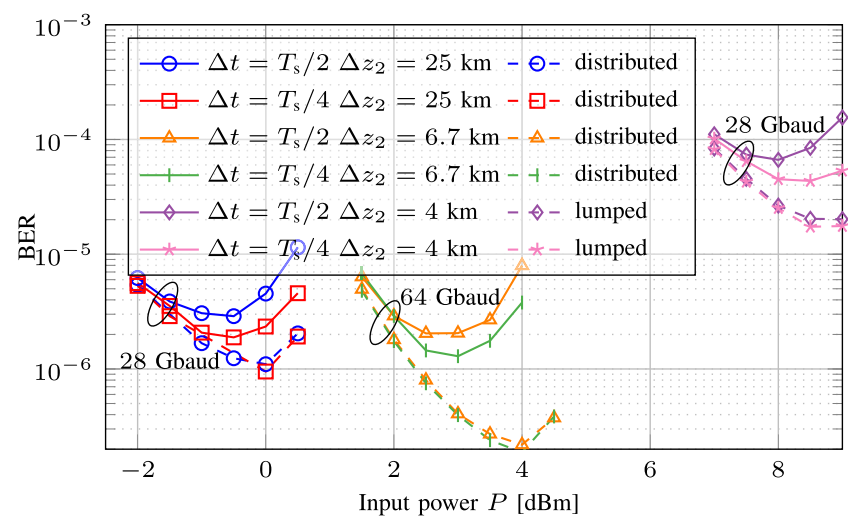

(a) 256-QAM

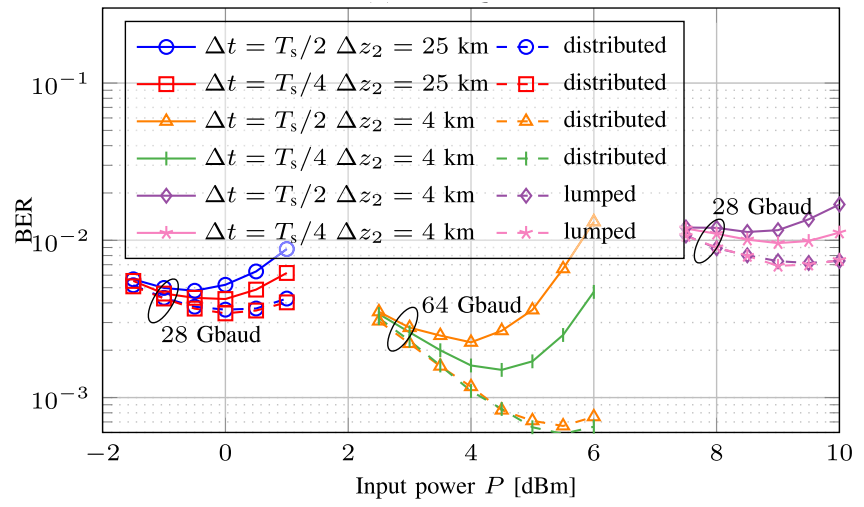

(b) 1024-QAM

Fig. 5. The BER is reduced with the LPFs in the TS-DBP for 256-QAM and 1024-QAM with 50\% lower DBP complexity. The lines with the same colors share the same parameters. The solid lines refer to the cases without LPFs, while the dashed lines refer to using LPFs.

$3.1 \times 10^{-6}$ to $1.1 \times 10^{-6}$, which is even lower than that with 4 samples $\left(2.1 \times 10^{-6}\right)$. At 64 Gbaud with 2 samples per symbol, the BER with LPFs is reduced from $2 \times 10^{-6}$ to $2.7 \times 10^{-7}$, which is even lower than with 4 samples $\left(1.3 \times 10^{-6}\right)$. Similar trends apply to $1024-\mathrm{Q} A M$ transmission, i.e., 2 times oversampling with LPFs outperforms 4 times oversampling. We also give an example with lumped amplification ${ }^{1}$ at 28 Gbaud, and show similar performance as distributed amplification. Thus, our modified DBP can effectively reduce aliasing and achieve simultaneously better performance and up to $50 \%$ complexity reduction compared with traditional DBP.

We also study the BER dependence on the filter bandwidth $W$ for the cases using distributed amplification with the lowest BER values, as illustrated in Fig. 6. When $W$ is around the baud rate, the BER is much higher than using standard all-pass filters, since the LPFs also remove useful signal information. The optimal $W$ which achieves the lowest BER depends on how strong the aliasing is. For example, in Fig. 6a, at 64 Gbaud, the optimal $W$ appears at around 1.5 times the baud rate both for 2 and 4 times oversampling. We illustrate the signal spectrum after the TS-DBP without LPFs for these two cases (marked with stars). When the LPFs filter out the the frequency noise above 1.5 times the baud rate, the lowest BER is achieved. Thus, the complexity of the linear step in

\footnotetext{
${ }^{1}$ Parameters: $K=3$ fiber spans of $L=100 \mathrm{~km} ; g(z)=\alpha L \sum_{k=1}^{K}$ $\delta(z-k L))$; noise figure $F_{n}=6 \mathrm{~dB}$; total ASE noise power:
} $2 K\left(e^{\alpha L}-1\right) F_{n} h v W_{\mathrm{s}}$. 


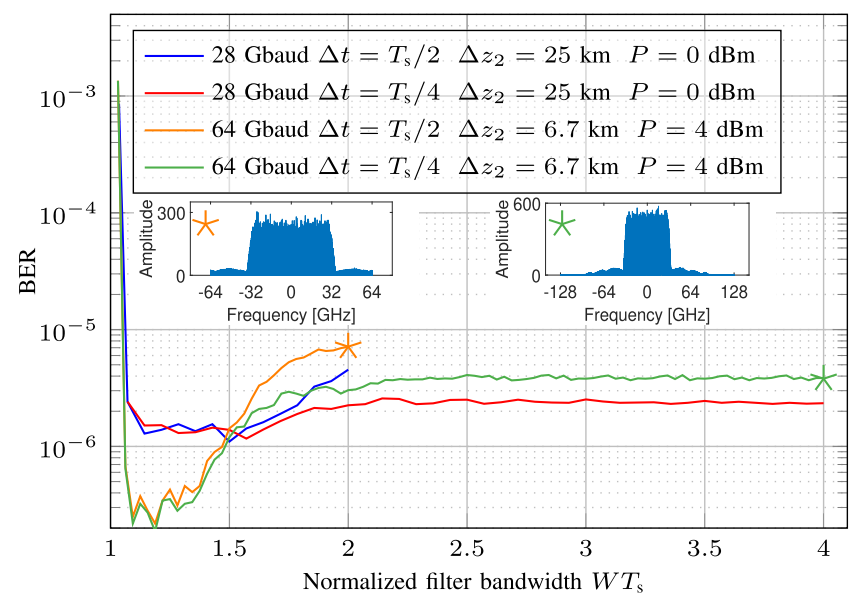

(a) 256-QAM

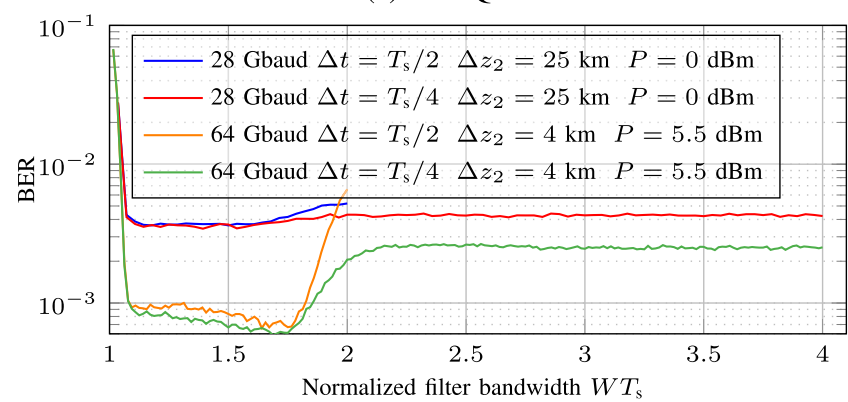

(b) 1024-QAM

Fig. 6. BER as a function of the filter bandwidth $W$ for 256-QAM and 1024-QAM. The optimal $W$ depends on the extent of aliasing. Parameters: $Z=1000 \mathrm{~km}$.

the DBP is reduced by around 55\%-70\% for the transmission with 4 samples per symbol using LPFs. Provided that $W$ is optimized, the power loss due to the LPFs also depends on the extent of aliasing, which is less than $3.5 \%$ in all cases in Fig. 6.

\section{CONCLUSION}

We proposed a modified aliasing-reduced SSFM algorithm with slightly reduced complexity by replacing the traditional all-pass filter with LPFs in the linear operator. A set of transmission cases with different system parameters such as modulation formats, baud rates, and oversampling rates were simulated. Our results show that 1) for forward propagation, the simulation error can be reduced with LPFs and 2) for TS-DBP, the complexity (the oversampling rate in the DBP) can be reduced by up to $50 \%$ with LPFs and the optimal bandwidth depends on the extent of aliasing. This filtering method allows for improved signal performance and reduced complexity both for the SSFM and the DBP. Although there is no universal answer to what value the optimal filter bandwidth should be, presimulations/experiments can always be done before the transmission/compensation given a SSFM/DBP complexity. In future work, further optimization of the filter parameters, such as the filter shape [10], [11], [18], could be considered to seek better performances.

\section{REFERENCES}

[1] R.-J. Essiambre and P. J. Winzer, "Fibre nonlinearities in electronically pre-distorted transmission," in Proc. 31st Eur. Conf. Opt. Commun. (ECOC), Glasgow, U.K., 2005, pp. 191-192.
[2] K. Roberts, C. Li, L. Strawczynski, M. O'Sullivan, and I. Hardcastle, "Electronic precompensation of optical nonlinearity," IEEE Photon. Technol. Lett., vol. 18, no. 2, pp. 403-405, Jan. 15, 2006.

[3] E. Temprana, E. Myslivets, B. P.-P. Kuo, N. Alic, and S. Radic, "Transmitter-side digital back propagation with optical injection-locked frequency referenced carriers," J. Lightw. Technol., vol. 34, no. 15, pp. 3544-3549, Aug. 1, 2016.

[4] E. Temprana et al., "Two-fold transmission reach enhancement enabled by transmitter-side digital backpropagation and optical frequency comb-derived information carriers," Opt. Express, vol. 23, no. 16, pp. 20774-20783, 2015.

[5] X. Li et al., "Electronic post-compensation of WDM transmission impairments using coherent detection and digital signal processing," Opt. Express, vol. 16, no. 2, p. 880, 2008.

[6] E. Ip, "Nonlinear compensation using backpropagation for polarizationmultiplexed transmission," J. Lightw. Technol., vol. 28, no. 6, pp. 939-951, Mar. 15, 2010.

[7] E. Ip and J. M. Kahn, "Compensation of dispersion and nonlinear impairments using digital backpropagation," J. Lightw. Technol., vol. 26, no. 20, pp. 3416-3425, Oct. 15, 2008.

[8] L. B. Du and A. J. Lowery, "Improved single channel backpropagation for intra-channel fiber nonlinearity compensation in longhaul optical communication systems," Opt. Express, vol. 18, no. 16, pp. 17075-17088, Aug. 2010.

[9] Y. Gao, J. H. Ke, J. C. Cartledge, K. P. Zhong, and S. S.-H. Yam, "Implication of parameter values on low-pass filter assisted digital back propagation for DP 16-QAM," IEEE Photon. Technol. Lett., vol. 25, no. 10, pp. 917-920, May 15, 2013.

[10] C. Häger and H. D. Pfister, "Nonlinear interference mitigation via deep neural networks," in Proc. Opt. Fiber Commun. Conf., San Diego, CA, USA, 2018, pp. 1-3.

[11] C. Hager and H. D. Pfister, "Deep learning of the nonlinear Schrödinger equation in fiber-optic communications," in Proc. IEEE Int. Symp. Inf. Theory (ISIT), Vail, CO, USA, Jun. 2018, pp. 1590-1594.

[12] D. Lavery, D. Ives, G. Liga, A. Alvarado, S. J. Savory, and P. Bayvel, "The benefit of split nonlinearity compensation for single-channel optical fiber communications," IEEE Photon. Technol. Lett., vol. 28, no. 17, pp. 1803-1806, Sep. 1, 2016.

[13] S. Musetti, P. Serena, and A. Bononi, "On the accuracy of split-step Fourier simulations for wideband nonlinear optical communications," J. Lightw. Technol., vol. 36, no. 23, pp. 5669-5677, Dec. 1, 2018.

[14] A. M. Heidt, "Efficient adaptive step size method for the simulation of supercontinuum generation in optical fibers," J. Lightw. Technol., vol. 27, no. 18 , pp. 3984-3991, Sep. 15, 2009.

[15] O. V. Sinkin, R. Holzlohner, J. Zweck, and C. R. Menyuk, "Optimization of the split-step Fourier method in modeling optical-fiber communications systems," J. Lightw. Technol., vol. 21, no. 1, pp. 61-68, Jan. 2003.

[16] Q. Zhang and M. I. Hayee, "Symmetrized split-step Fourier scheme to control global simulation accuracy in fiber-optic communication systems," J. Lightw. Technol., vol. 26, no. 2, pp. 302-316, Jan. 15, 2008.

[17] J. Shao, X. Liang, and S. Kumar, "Comparison of split-step Fourier schemes for simulating fiber optic communication systems," IEEE Photon. J., vol. 6, no. 4, pp. 1-15, Aug. 2014.

[18] Y. Zhu and D. V. Plant, "Optimal design of dispersion filter for timedomain split-step simulation of pulse propagation in optical fiber," J. Lightw. Technol., vol. 30, no. 10, pp. 1405-1421, May 15, 2012.

[19] J. Garcia, H. Ghozlan, and G. Kramer, "Energy conservation in optical fibers with distributed brick-walls filters," J. Lightw. Technol., vol. 36, no. 9, pp. 1626-1633, May 1, 2018.

[20] S. Li, M. Karlsson, and E. Agrell, "Improved simulation accuracy of the split-step Fourier method," in Proc. Opt. Fiber Commun. Conf. (OFC), San Diego, CA, USA, 2020, p. W2A-55.

[21] E. Agrell, "Capacity bounds in optical communications," in Proc. Eur. Conf. Opt. Commun. (ECOC), Gothenburg, Sweden, Sep. 2017, pp. 1-3.

[22] K. Keykhosravi, "Capacity analysis and receiver design in the presence of fiber nonlinearity," Ph.D. dissertation, Dept. Elect. Eng., Chalmers Univ. Technol., Gothenburg, Sweden, 2019.

[23] Y. R. Shen and G.-Z. Yang, "Theory of self-Phase modulation and spectral broadening," in The Supercontinuum Laser Source, R. R. Alfano, Ed. New York, NY, USA: Springer, 1989.

[24] G. P. Agrawal, Lightwave Technology: Telecommunication Systems. Hoboken, NJ, USA: Wiley, 2005.

[25] R.-J. Essiambre, G. Kramer, P. J. Winzer, G. J. Foschini, and B. Goebel, "Capacity limits of optical fiber networks," J. Lightw. Technol., vol. 28, no. 4, pp. 662-701, Feb. 15, 2010. 